\title{
Design of Micro-lecture for College Chemistry Majors and Prospect of Its Teaching Application
}

\author{
Sun Linhao
}

\author{
College of Science, Huazhong Agricultural University, Wuhan 430070, China
}

Keywords: Internet; educational mode; chemistry specialty; micro-lecture

\begin{abstract}
With the continuous development of network technology, the education has followed the pace of the times and carried out a series of educational model reforms on the basis of the Internet. There are various aspects to be improved in the traditional teaching mode of the chemistry specialty in colleges and universities. But after the introduction of "micro-lecture", the teaching mode has changed and the teaching quality has also been significantly improved. By analyzing the current situation of the teaching mode of chemistry specialty in Chinese universities, the author proposed to apply the "micro-lecture" in the teaching of chemistry majors and explored how to design the "micro-lecture" for the courses of chemistry specialty in universities.
\end{abstract}

\section{Introduction}

This is an Internet age and most college students now in China were born after 1995. They are nurtured in the Internet age and prefer to pursue modern high-tech new things. The traditional teaching mode is no longer suitable for contemporary college students, so a number of schools have launched "micro-lecture" relying on Internet technology. It is a new educational method integrating teaching plans, courseware, exercises and videos, and its most prominent features are visualization, digitalization and convenience $^{[1]}$. It is also welcomed by schools and students. In this sense, to discuss the design and application of micro-lecture in the teaching for chemistry majors is necessary in the reform of teaching mode of chemistry specialty in modern colleges and universities.

\section{The Definition and Origin of Micro-Lecture}

As the name implies, "micro-lecture" is a short lesson that takes the advantage of pictures, words and sounds to show the teaching content. It mainly takes short videos as its carrier to record teachers' explanation of key points, difficulties and test points from teaching contents in class. It broke the traditional teaching mode and has formed a new teaching mode with courseware segments as its core ${ }^{[2]}$. With clear teaching objectives and short and concise contents, micro-lecture conforms to the requirements of the new curriculum and it has become the inevitable trend of the development of modern education in the Internet age. In 1960, the University of Iowa first proposed the concept of micro-lecture, which is also called as "short-term course” or "modular course”. In 2008, “one-minute micro video" began to spread in the United States and received wide attention, with the purpose of providing students with clear knowledge. Micro-lecture, which was first used in the field of chemistry teaching, has the advantages of distinct theme, diverse content, real situation, enhanced communication and convenient use ${ }^{[3]}$.

\section{Advantages of Micro-Lecture}

Micro-lecture is characterized by being short and pithy, allowing students to arrange independent study for themselves in their spare time, which has aroused countless students' love for it. With its emergence, students can use mobile devices such as tablet computers and mobile phones to learn anytime and anywhere. At the same time, the content of short videos focuses on error-prone language points, difficulties, exam hints and exercises of knowledge, which greatly improves students' learning efficiency and facilitates them to review. Some specific features of micro-lecture are as follows. 


\subsection{Short video time}

The main carrier of micro-lecture is the short video, which usually lasts five to ten minutes. Students can make use of their spare time to study, and furthermore, the short-time study can make their attention highly concentrated. In teaching, teachers should make full use of the real classroom and bring the introduction, teaching emphasis, example explanation as well as after-class exercises and review into the real classroom. However, because the time is very limited, the teachers must master the teaching content and process skillfully.

\subsection{Tailored contents}

Videos of micro-lecture are mainly about a certain knowledge points. Because the time of a video is very short, it contains few but highly-targeted contents. In the process of teaching, teachers should highlight the key points, difficulties, error-prone language points and exam hints. It is also necessary to master multimedia teaching techniques skillfully and enable students to make full use of teaching techniques for autonomous learning, so as to achieve better educational results. However, it is particularly important to note that micro-lecture is also a lesson. Although it is short, it still cannot ignore the basic teaching steps of a whole lesson.

\subsection{Various learning channels}

Micro-lecture is a short-time video teaching with concentrated contents implemented by Internet technology. It supports online play, which satisfies the demand of teachers and students. In addition, it can be downloaded, reprinted and saved so that students and teachers can learn independently. Its support for distance learning and mobile learning has greatly increased the communication between teachers and students.

\subsection{Realization of one-on-one teaching}

The content of micro-lecture is highly targeted, so it actually can be regarded as a kind of one-on-one teaching. It can not only be watched online, but can also be replayed, which improves students' autonomous learning ability and the learning effect. After class, students can review and address gaps through micro-lecture to achieve cooperative communication with their classmates [5]. Micro-lecture is not only good for students but also benefits teachers, because it provides teachers with a lot of tutorial resources. In the Internet era of resource sharing, the development of micro-lecture has enabled different teachers throughout the country to achieve cross-regional exchanges and enrich their teaching modes through learning and innovation.

\subsection{Creating teaching situations}

Micro-lecture is a new teaching mode integrating text, picture and sound, which completely reflects the teaching situation in the classroom. Although the time of a video is short, the teaching resources that micro-lecture covers are extremely rich, which can become the main channel for students to review after class and improve their autonomous learning ability. The communication between teachers and students, as well as students' feedback can also be reflected in micro-lecture. Together with teaching videos, these contents constitute a resource-rich teaching resource package.

\section{The Present Situation of College Chemistry Teaching in China}

With the continuous development of China's economy, the market proposes increasing demand for chemical professionals, leading to an increase in the number of university admissions each year. For students of a university from all parts of the country, the overall level of their basic knowledge is not uniform when they enter the university due to the different educational levels in the region they are educated. After entering the university, the traditional teaching mode of chemistry courses is for all students, which makes it very difficult for students with poor foundation to follow up. In addition, the traditional teaching mode of chemistry is based on the teacher's teaching of knowledge, and the experiment teaching is also based on the teacher's demonstration on the platform, which greatly reduces the enthusiasm of students to participate in the classroom and makes them not interested in chemistry ${ }^{[6]}$. Some colleges and universities have set up experimental courses in chemistry teaching, but due to the 
lack of complete equipment, many experiments are only at the theoretical level and cannot realize the training of students' practical ability.

\section{Design Rules of Micro-Lecture}

\subsection{Short but pithy}

Micro-lecture is characterized by being short and concise, and a video of the micro-lecture generally lasts less than ten minutes. Therefore, educators should grasp the course introduction, explanation of key language points, example analysis, final course summary of exercise for a micro-lecture, as well as the time length of each links. To make micro-lecture "capable and vigorous", educators must highlight the main body of the teaching content, decompose the difficulties one by one and find the inner connection of knowledge, so that students can easily understand the educators' explanation and thinking.

\subsection{Vivid and interesting}

A micro-lecture video generally focuses on one knowledge point, so the content must be short, concise, easy to understand, lively and interesting ${ }^{[7]}$. Teachers should pay attention to the consistency of animation picture, character color, image and sound in videos when creating micro-lecture, so as to attract students' attention and improve their interest in learning by reasonable deployment through software technology.

\subsection{Complete and shared}

Although a video of micro-lecture is short, it still contains a complete teaching process, namely, the introduction of the lesson, the explanation of key knowledge points, the analysis of examples, the explanation of exercises and the summary of this lesson. The teacher must make good use of each link to highlight the teaching content and enhance students' learning efficiency. At the same time, micro-lecture can be spread through the network to realize resource sharing, which facilitates students' autonomous learning anytime and anywhere.

\section{Design Process of Micro-Lecture}

\subsection{Select the content and theme}

Content and topic selection is an important part of micro-lecture, and thus teachers should be able to select typical knowledge points as materials for micro-lecture. Because videos of micro-lecture are short in time, they are supposed to present the teaching contents vividly and interestingly so as to attract students' attention and improve their learning. Choosing key and difficult language points as the content to be explained can strengthen students' understanding of these points, and the choice of the theme is also to lay the foundation for designing teaching plans.

\subsection{Design teaching plans}

The teaching plan design for micro-lecture should take into account many aspects, such as the teaching objective, teaching strategy, students' characteristics, and so on. The specific process of teaching covers leading-in, sorting out contents, inserting tasks and summarizing. The leading-in is often an interesting question to arouse students' attention and interest at the beginning of the short class and to introduce the explanation of the class. Sorting out the content is to sort out the collected data and find out the important and difficult knowledge points to focus on. Inserting tasks is to release a task after the knowledge explanation, with the purpose to check whether the students have mastered the explained knowledge points. Summary is to make a summary of this lesson just before the end of video to help students systematically master the knowledge point.

\subsection{Make courseware}

The videos of micro-lecture must contain exquisite courseware for teaching and the software Power Point is often used to make courseware. Each PPT should have exquisite, brief and intensive contents. Pictures and characters on PPT should keep a unified style, because colors too complicated will lead to students' inattention. Furthermore, in addition to words, PPT must contain pictures or animations to enhance students' interest in learning. 


\subsection{Record video}

The final step of making micro-lecture is recording the video. When recording a video, PPT made previously should be used in the process of explanation and the explanation must fully conform to the content order made in the PPT. Only in this way can students' thinking keep up with the teachers' and improve their learning efficiency.

\section{Advantages of Micro-Lecture Integrating into Courses for Chemistry Majors}

\subsection{Giving full lay to students' subjectivity}

At present, the major courses for chemistry majors in Chinese universities mainly adopt traditional teaching modes. Most chemistry experiments are conducted in the classroom. Students can only carry out the relevant operations under the guidance of their teachers and are not allowed to enter the laboratory for operation and study without permission, which results in students being unable to use their effective time to select and study the specialty that they are interested in. However, the emergence of micro-lecture make students is able to learn professional knowledge that they are interested in anytime and anywhere. They can communicate with teachers online even though they do not know professional knowledge well, which gives full play to their subjectivity in learning and improves their ability of autonomous learning. Through micro-lecture, students now can easily and intuitively learn the process of many experiments that they could only watch in the laboratory in the past, which makes learning more integrated into their daily life.

\subsection{Improving students' learning efficiency}

Under the traditional teaching mode of chemistry courses, students cannot visually and clearly observe the process of teachers doing experiments in class due to the limitation of equipment conditions. Most teachers also omit this practical operation process and only teach theory to enable students to master knowledge, which is not conducive to students' enthusiasm for learning. With the emergence of micro-lecture, students can learn intuitively through short videos and can also choose contents they are interested in. They can fully grasp the knowledge points they need to learn by watching the teacher's experimental operation and explanation in the relevant video, greatly improving the students' learning efficiency.

\subsection{Promoting teachers' professional development}

The teaching content of micro-lecture comes from the difficulties that students cannot understand in the learning process, and some strategies adopted by teachers in teaching are combined together to edit the teaching script of micro-lecture. In the creation process of micro-lecture, teachers should pay attention to the teaching process, promote the communication among teachers, and imperceptibly cultivate their own ability of unity and cooperation. The creation of micro-lecture has transformed in-class teaching into out-of-class teaching, not only liberating teachers but also improving teaching quality.

\subsection{Optimizing teaching resources of chemical experiments}

Experimental courses are very important for students majoring in chemistry at university, but the traditional teaching mode results in students' insufficient ability to operate in practice. Micro-lecture is a kind of videos that can be shared online. It can optimize and integrate the teaching materials of chemical experiments so that students can fully and clearly understand the operation procedures and precautions of chemical experiments, helping them lay the foundation for the following courses ${ }^{[8]}$.

\subsection{Adapting to information age}

In this information age, the information people are exposed to be complicated, but their time is limited. How can people get access to effective information in a limited time? The characteristics of micro-lecture determine its success. It is a new way of learning which is short in production time, concise in content and flexible in operation. It well meets the requirements of college students for short-time information and effective contents, and it also adapts to this information age. 


\section{Conclusion}

The introduction of micro-lecture into the college chemistry courses and its integration with the traditional model can provide targeted education for students with different knowledge basis, which is conducive to improving the learning quality of chemistry majors. However, colleges and universities cannot use micro-lecture as the only method to teach because it is too short to explain some little knowledge details. It can only be used as an auxiliary tool of the traditional education to help students review in time and make up their deficiencies. Only by combining the traditional education mode with micro-lecture can the reform and development of chemistry education in colleges and universities be facilitated.

\section{Acknowledgment}

Fund project: National Natural Science Foundation of China (No. 21502058)

\section{Reference}

[1]. Liang Leming, Cao Qiaoqiao, Zhang Baohui. Research on a Micro-lecture Design Model through Comparative Case Study [J]. Education Research 2013 (1): 65-73

[2]. Du Hui, Zhang Yiping, Wang Shuhong, et al. The Application of Micro-Course in Chemistry Experiment Teaching [J]. Journal of Anyang Institute of Technology 2014 (6): 102-105

[3]. Ma Hadan. Application of Flipped Classroom Model based on Micro-lecture in College English in Independent Colleges [J]. Overseas English. 2016 (21): 86-87

[4]. Zhang Shuyong, Zhang Jianrong, Chen Liuping. On the Problems and Reform of Undergraduate Chemistry Laboratory Teaching [J]. University Chemistry. 2009, 24 (4): 24-28

[5]. Meng Jing, Hong Yanfen. Analysis of the Application of Micro-Lecture in Chemistry Teaching -Taking the Micro-Lecture Teaching of "Electrolytic Saturated Salt Water" as an Example [J]. Education in Chemistry. 2016 (12): 37-40

[6]. Song Jinfan, Guo Xinfeng, Wang Shengzhao, et al. Application of Micro-lecture in the University Physics Experiment Teaching [J]. Physics Experiment. 2015 (2): 12-17

[7]. Chen Fei, Wang Zhiyou, Chen Lin. et al. Application and Practice of Micro-lecture Teaching Mode in "Buffer Solution" Course [J]. Journal of University of Science and Technology Liaoning. 2013 (4): 36-37

[8]. Hu Tiesheng, Huang Mingyan, Li Min. The Three Stages of Micro-lecture Development and Its Enlightenment [J]. Distance Education Journal. 2013 (4): 36-37 